\title{
Comparative Study on Acellular Dermal Graft Versus Propylene Mesh Both Either Loaded or Unloaded with BM-MSCs in Healing of Skull Bone Defect in Rats: Histological and Immunohistochemical Study
}

\author{
Lobna Elkhateb ${ }^{1}$, Adel Zohdy ${ }^{1}$, Suzi Sobhy Atalla ${ }^{1}$, Manal Hassan Moussa ${ }^{1}$, \\ Ghada Galal Hamam', Fatma Abd Elkarim Abu Zahra ${ }^{2}$ \\ ${ }^{1}$ Department of Histology and Cell Biology, Faculty of Medicine Ain Shams University, Cairo, Egypt \\ ${ }^{2}$ Medical Research Center, Ain Shams University, Cairo, Egypt
}

\begin{abstract}
Bone defect occurs as a consequence of many conditions. Diseased bones don't heal properly and defects in face area need proper bone reconstruction to avoid psychological and social problems. Tissue engineering is an emerging new modality of treatment. We thought to study different methods to fill skull bone defect in rats in order to find the most safe and effective method. So, this study was designed to evaluate the efficacy of acellular dermal graft (ADM) versus propylene mesh both either loaded or unloaded with bone marrow derived mesenchymal stem cells (BM-MSCs) in healing of skull bone defect of a $5 \mathrm{~mm}$ diameter. The study included 36 adult male Wistar albino rats that were divided into three groups according to the way of filling skull bone defect. Group I: Ia (sham control), Ib (negative control). Group II: IIa (unseeded propylene), IIb (seeded propylene) and Group III: IIIa (unseeded ADM), IIIb (seeded $\mathrm{ADM})$. The trephine operation was done on the left parietal bone. Specimens were collected four weeks postoperative and processed for H\&E, osteopontin immunohistochemistry and scanning electron microscope. Morphometric and statistical analysis were also performed. After studying the results of the experiment, we found that propylene mesh and $\mathrm{ADM}$ were suitable scaffolds that could support new bone formation in clavarial bone defect. Healing of skull bone defect was better in rats that received seeded scaffolds more than rats with unseeded scaffolds. The seeded ADM showed significant increase in bone forming activity as confirmed by histomorphometric and statistical results.
\end{abstract}

Keywords: Bone defect, Propylene mesh, Acellular dermal graft, Histology, Bone marrow derived mesenchymal stem cells, Immunohistochemical

Received: March 4, 2018, Revised: April 17, 2018,

Accepted: April 23, 2018, Published online: August 31, 2018

Correspondence to Ghada Galal Hamam

Department of Histology and Cell Biology, Faculty of Medicine Ain Shams University, Abbassyia, Cairo 11566, Egypt

Tel: +201003960601, Fax: +20 223496662

E-mail: ghada.hamam@yahoo.com

(c) This is an open-access article distributed under the terms of the Creative Commons Attribution Non-Commercial License (http://creativecommons.org/ licenses/by-nc/4.0/), which permits unrestricted non-commercial use, distribution, and reproduction in any medium, provided the original work is properly cited.

Copyright (c) 2018 by the Korean Society for Stem Cell Research

\section{Introduction}

When bone is injured, dramatic changes occur in the quality of life of patients. It frequently causes social and psychological problems (1). Critical-sized bone defect was defined as the smallest intra-osseous defects in a specific bone that do not heal spontaneously in the lifetime of the animal (2).

About 2.2 million people worldwide undergo bone grafting due to critical-sized bone defects (3). As an alternative to autologous or allogeneic bone grafts, new approach of bone-tissue engineering has emerged. Surgeons use scaf- 
folds made of synthetic or natural biomaterials that promote the migration, proliferation, and differentiation of bone cells. Scaffolds can be loaded with osteogenic cells in order to generate a living bone graft in vitro, and thus improve clinical outcome (4).

Polypropylene is one of the most inert biomaterials used in surgery, and is available as a woven mesh. It is has good tensile strength, and demonstrates early fibrous tissue ingrowth that serves to fix and incorporate the mesh. Acellular dermal graft (ADM) is structurally and biochemically intact implant material. It is mainly formed of collagen; the most common protein in the body. The graft serves as a framework to support cellular repopulation, revascularization at the surgical site, and soft tissue regeneration by the recipient's own cells (5).

To provide osteoinductivity, scaffold materials can be loaded in vitro with mesenchymal stem cells (MSCs) in order to generate a living bone graft (6). Osteopontin is a protein that could be found in both extra cellular matrix (ECM) and cytoplasm of some cells. It is also produced by endothelial cells and vascular smooth muscle cells. It is an inflammatory mediator secreted by inflammatory cells (7).

\section{Aim}

To evaluate the efficacy of ADM versus propylene mesh either loaded or unloaded with BM-MSCs in healing of $5 \mathrm{~mm}$ skull bone defect in adult male albino rats.

\section{Materials and Methods}

\section{Animals}

Forty six male Wistar albino rats were used in this study. The experiment was conducted on 36 adult rats each weighing 150 200 gms. Their ages ranged from two to three months. Five rats of the same age and weight were used to obtain ADM. Five weaned male rats weighing 70 80 gms each and their ages around one month, were used for preparation of BM-MSCs. Animals were kept under proper conditions of light, temperature and humidity. The whole experiment was carried out in the Animal Research Center, faculty of medicine, Ain Shams University.

All animal procedures were carried out according to the guideline of animal care and the scientific research ethical committee of the faculty of Medicine, Ain Shams University.

\section{Experimental protocol}

Animals were kept for seven days before beginning the experiment for acclimatization. The 36 experimental rats were randomly divided according to the way of filling skull bone defects into three equal groups, 12 animals each. Each group was further subdivided into two subgroups six animals each. Surgical operation was carried out on all rats, except those for sham control.

\section{Group I: Control group}

- Subgroup Ia: (sham control): rats were left without any interference.

- Subgroup Ib: (negative control): skull bone defect were left to heal spontaneously.

\section{Group II: Synthetic mesh group}

- Subgroup IIa (unseeded propylene mesh): skull bone defects were filled with propylene mesh only.

- Subgroup IIb (seeded propylene mesh): skull bone defects were filled with propylene mesh loaded with BM-MSCs.

Group III: Dermal graft group

- Subgroup IIIa (unseeded ADM): skull bone defects were filled with ADM only.

- Subgroup IIIb (seeded ADM): skull bone defects were filled with ADM loaded with BM-MSCs.

After one month from the operation, all animals were sacrificed.

\section{Surgical procedure}

Preoperative preparation: Animals were anesthetized by intramuscular injection of xylazine/ketamine combination (8). Preoperative dose of sterile normal saline was given by intraperitoneal injection based on $10 \mathrm{ml} / \mathrm{kg} / \mathrm{hr}$ of surgery. Skulls of rats were shaved from the bridge of the snout between the eyes to the caudal end of the skull. Then an alcohol swab was used to remove hair trimmings (9).

Operative procedures: Operative procedures were done as previously described (9). The trephine tool measured $5 \mathrm{~mm}$ in diameter. The margins of the defects were intended to be away from skull sutures (Fig. 1A). In animals of group II and III, the implant material (ADM or propylene mesh) was placed into the defect (Fig. 1B). The skin was closed using simple interrupted 2-0 nylon suture. The head was cleaned with saline and was sprayed with amikacin antibiotic.

Postoperative care: After observation of purposeful movements, rats were transferred to their normal cages and were housed singly until tissue harvest. Betadine and Antibiotic spray were applied to the surgical site for three days postoperative. All 36 rats received intraperitoneal injections of analgesic and antibiotic for one week postoperative. 

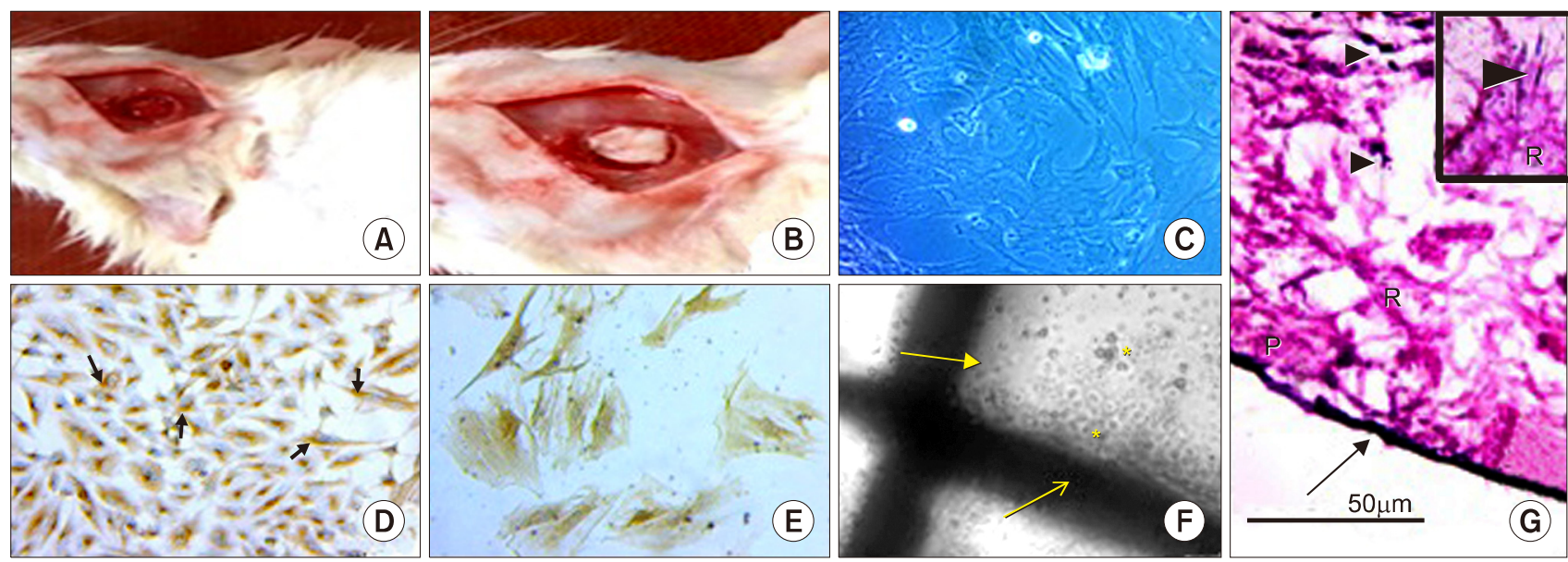

Fig. 1. (A) The defect after trephine. (B) ADM in the defect. (C E) Phase contrast photomicrographs of MSCs showing (C) Sub-cultured MSCs on day 5. Most cells are spindle in shape with long cytoplasmic processes having granular cytoplasm with vesicular nuclei. (D\&E) Cells of primary culture showing MSCs with positive brownish cytoplasmic immune reaction for CD $105(\uparrow)$ and CD44 respectively. (F) Propyelene mesh fibers $(\uparrow)$ in culture media with BM-MSCs $(*)$. (G) H\&E of ADM after seeding with BM-MSCs. Irregular collagen fibers in papillary $(P)$ and reticular $(R)$ layers are covered with basement membrane $(\uparrow)$. Inset: Cells $(\boldsymbol{\Lambda})$ between collagen fibers. ADM: acellular dermal graft, BM-MSCs: bone marrow derived mesenchymal stem cells. Phase contrast photomicrographs [C $\times 200],[D \times 100],[E \times 400]$. H\&E [G×400, inset $\times 1,000]$.

\section{Implant materials}

Prolene meshes (10): Polypropylene knitted non-absorbable, sterile mesh was purchased from EL Goumhoria company (Ethicon, INC, Trademark RMC: 385972). The mesh was cut to fit into the defect area.

Acellular Dermal Graft: Decellularization of the skin was done to prepare ADM. The skin was treated to remove the cells of the epithelial and dermal components. It was prepared by combination of physical and chemical decellularization techniques $(11,12)$.

Before use, the graft was sterilized by exposure to UV rays (two hours for each side) (11). A specimen of $1 \mathrm{~cm}$ $\times 1 \mathrm{~cm}$ of the prepared dermis was then fixed in $10 \%$ buffered formalin and processed to obtain paraffin blocks. Paraffin sections were then stained with H\&E stain.

\section{Culture and isolation of BM-MSCs}

Isolation, culturing and characterization of cultured BM-MSCs were done (13). All steps were carried out at Stem cell research unit, Histology and Cell Biology department, Faculty of Medicine, Ain Shams University. Immunohistochemical characterization of cultured BM-MSCs was done using streptavidin-biotin immunoperoxidase technique for CD44, CD 105, CD 45 and CD 34 in the cultured cells (14).

\section{Seeding of BM-MSCs on ADM and propylene mesh}

Round pieces of $\mathrm{ADM}$ ( $5 \mathrm{~mm}$ diameter) were prepared.
After sterilization, $\mathrm{ADM}$ was soaked in saline for one hour to rehydrate it. BM-MSCs were obtained near confluent in the $2^{\text {nd }}$ passage. $A D M$ scaffolds were incubated overnight in fresh MSCs Growth Medium. $0.5 \mathrm{ml}$ of BM-MSCs suspension containing $1 \times 10^{5}$ MSCs was pipette aseptically onto ADM. It was left for one hour in the laminar flow cabinet to help cell attachment then $2 \mathrm{ml}$ of Dulbecco's modified Eagles medium was added. For transplantation onto animal, these scaffolds were cultured for one day after seeding with MSCs (15).

The same steps were applied to seed BM-MSCs on propylene mesh.

\section{Sample collection}

At the end of the experiment, animals were sacrificed by decapitation after ether inhalation anesthesia. The surgical site surrounded with an area of healthy clavarial bone was collected.

\section{Preparation of tissue}

Preparation of decalcified material: The collected samples were decalcified using ethylene-diamine-tetraacetic acid (EDTA) disodium salt. The decalcified specimens were processed to paraffin blocks. Serial sections were cut in a plane perpendicular to bone surface and were stained with H\&E and streptavidin-biotin immunoperoxidase technique for detection of osteopontin (Lab vision/Neomarkers. Westinghouse Dr. Fremont, California) 
(16). Osteopontin is also called secreted phosphoprotein 1 (SPP1). Osteopontin active sites appear brown both in the cytoplasm of cells and in the extracellular matrix (ECM). Negative control was done by omitting the step of primary antibody.

Preparation for scanning electron microscopy (SEM): Area of defect surrounded by healthy clavarial bone was taken from all groups. The specimens were cut in plane perpendicular to the bone surface, and then processed for SEM. Specimens were examined and photographed using a JEM 1200 EXII (JEOL, Tokyo, Japan) SEM at Faculty of Science, Ain Shams University. Cairo, Egypt.

\section{Morphometric and statistical study}

An image analyzer Leica Q win V.3 program installed on a computer in the Department of Histology and Cell Biology, Faculty of Medicine, Ain Shams University, was used. The computer was connected to a Leica DM2500 microscope (Wetzlar, Germany).

Animals from all groups were subjected for morphometric study. Measurements were taken from three different slides obtained from each animal. Five haphazardly selected non overlapping fields were examined for each slide at objective lens $\times 40$ to measure.

Percentage of bone surface covered by osteoblasts: It represented the percentage of bone perimeter covered by osteoblasts.

The mean area percentage for positive expression of osteopontin: Mean and standard deviation (SD) of measured parameters were statistically analyzed using one way ANOVA with post-hoc test using SPSS statistical program version 21 (IBM Inc., Chicago, Illinois, USA). Probability of $\mathrm{p}$ value $<0.05$ is considered significant (17).

\section{Results}

The morphology of the primary culture of BM-MSCs by the inverted phase contrast microscope

On examination of the BM primary culture on day three, some cells were seen adherent to the flask. They were variable in size and shape. They appeared spindle, star shaped and triangular. The cells showed vesicular nuclei. On day four, some cells appeared rounded, spindle in shape while others were star shaped. They attained vesicular nuclei and granular cytoplasm. Some cells had long well developed processes. Colony formation could be also noticed. On day seven the cells were mostly confluent (about 90 95\%) with granular cytoplasm, vesicular nuclei and long interconnected cytoplasmic processes (data not shown). The rat BM-MSCs on day five of subculture reached about 90 95\% confluence and was formed of colonies of spindle shaped cells (Fig. 1C). At this stage they were collected for seeding on scaffolds.

Immunohistochemical examination of cells of primary culture showed positive CD105 and CD44 brownish cytoplasmic reaction of attached cells (Fig. 1D and 1E respectively). Also negative immune reaction for CD34 and CD45 was noticed (data not shown).

\section{Results of seeding of BM-MSCs on scaffolds}

After seeding on propylene mesh, examination of the culture dishes by phase contrast microscope, revealed propylene mesh as squares of fibers and BM-MSCs were seen seeded on it (Fig. 1F). After seeding on ADM; cells were seen between collagen fibers (Fig. 1G).

\section{Histological results}

In subgroup Ia, examination of sagittal sections of the cranial vault stained with $H \& E$, showed the clavarial bone formed of outer and inner tables of compact bone with diploe formed of BM spaces separated by bone trabeculae. The outer surface of the compact bone was covered with periosteum and the inner surface was seen lined with dura matter (data not shown). BM spaces were lined with endosteum containing flat osteogenic cells and cuboidal osteoblasts. Osteocytes were seen inside their lacunae. Basophilic cement lines could also be noticed between parallel bone lamellae (Fig. 2A).

In subgroup $I b$ the bone defect was filled with dense irregular vascular connective tissue (CT). Blood vessels were noticed in the CT and few osteogenic cells were seen at the margin of the bone defect. Small foci of newly formed bone with uneven acidophilic staining of the matrix were sometimes seen near the margin of bone defect (Fig. 2B). Osteoblasts were occasionally noticed on the surface of bone. Fibroblasts with flattened nuclei were frequently seen between bundles of densely arranged collagen fibers. Many blood vessels could be also seen between the collagen fibers (Fig. 2C). Some blood vessels were seen at the center of the defect surrounded by mononuclear cells including some lymphocytes with large dark basophilic nuclei and plasma cells with eccentric cart wheel nuclei and negative Golgi image (Fig. 2D).

In subgroup IIa the defect area was filled with BM. Thin rim of bone was seen on the outer and inner surfaces of the defect with some attached cells. Osteogenic cells and osteoblasts were seen on the margin of the bone at the defect area (Fig. 3A). Osteogenic cells were also seen in the inner osteogenic layer of the periosteum (data not shown). 

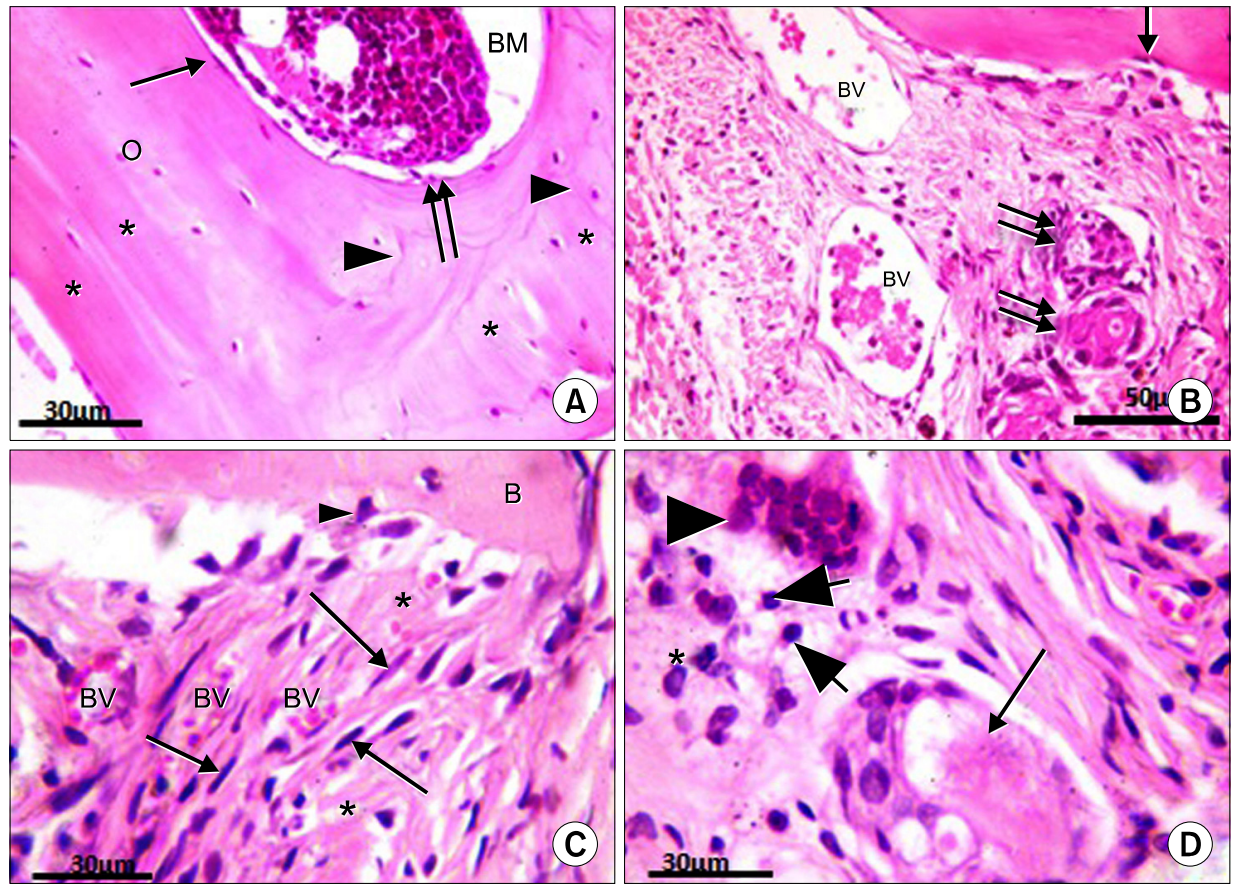

Fig. 2. (A) la: Clavarial bone formed of diploe of compact bone with bone marrow space (BM) in-between. Endosteal surface lined with osteogenic cells $(\uparrow)$ and osteoblasts $(\uparrow \uparrow)$, osteocytes inside their lacunae $(\mathrm{O})$, and basophilic cement lines $(\mathbf{\Lambda})$ are seen between bone lamellae ${ }^{*}$ ). (B D) Ib: (B) Bone defect filled with dense vascular (BV) connective tissue, few osteogenic cells $(\uparrow)$ at the margin of the defect, small foci of newly formed bone $(\uparrow \uparrow)$. (C) Richly vascularized (BV) dense connective tissue in bone defect, fibroblasts $(\uparrow)$ between bundles of densely arranged collagen fibers $(*)$. Occasional osteoblasts $(\mathbf{A})$ on bone surface (B). (D) A blood vessel is surrounded with homogenous acidophilic material $(\uparrow)$, mononuclear cells $(\boldsymbol{\Lambda})$, lymphocytes (thick arrow) and plasma cells $(*)$. H\&E [A, C\&D] $\times 1,000$. [B] $\times 400$.
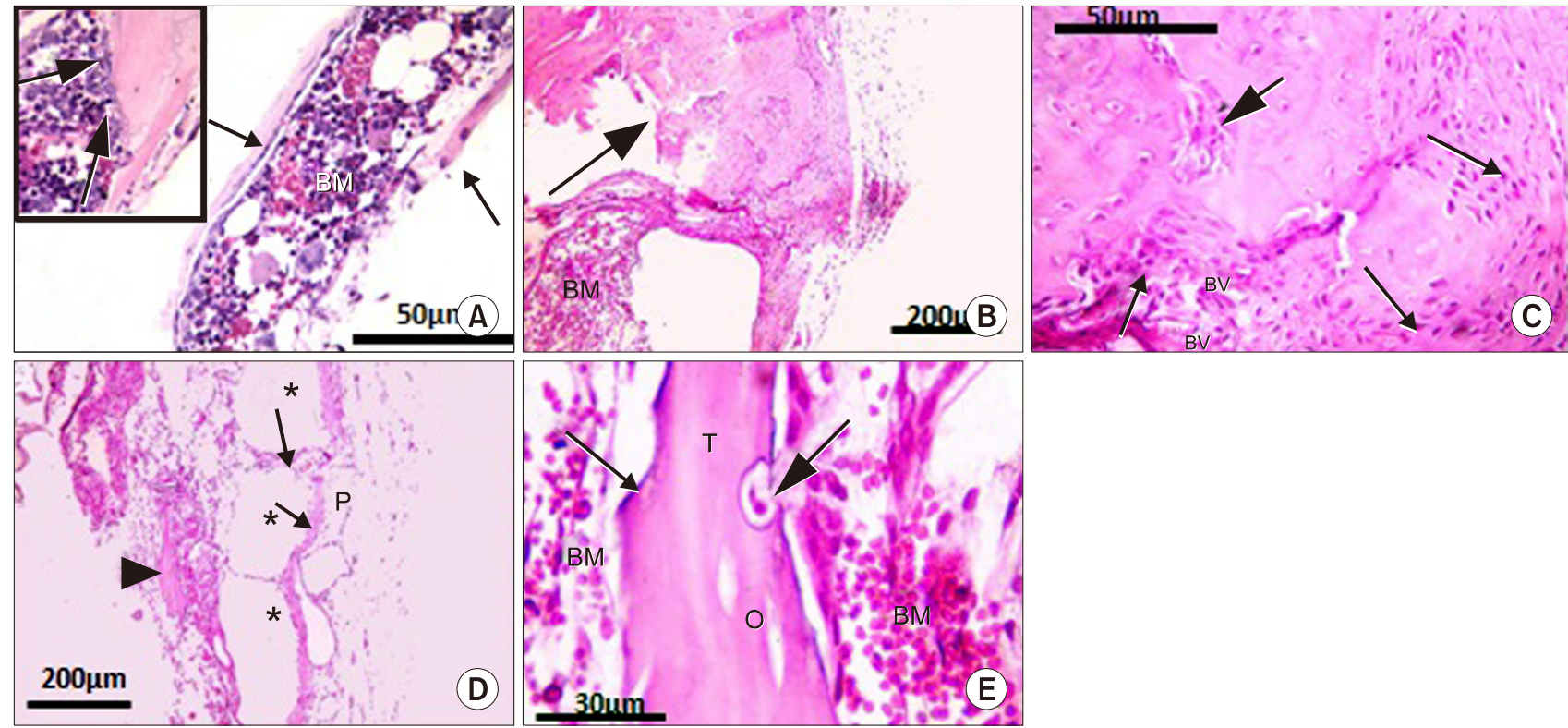

Fig. 3. (A) Ila: Thin rim of bone $(\uparrow)$ with attached cells on inner and outer surfaces of defect with bone marrow (BM) inbetween. Inset: margin of defect with osteogenic cells (thick arrow). (B E) $I / b$ : (B) Irregular immature bone $(\uparrow$ ) and BM space near the defect. (C) Highly celluar bone margin, osteocytes in their lacunae, osteoblasts $(\uparrow)$, blood vessels (BV) and irrregulaly formed bone (thick arrow) near defect margin. (D) Bone defect filled with many wide spaces $\left(^{*}\right)$ separated by thin septa $(\uparrow)$ and covered with periosteum (P). Area of new bone formation is seen $(\mathbf{\Delta})$. (E) Newly formed bone trabecula $(T)$, oesteogenic cells $(\uparrow)$, osteocyte lacunae $(\mathrm{O})$, an osteoclast (thick arrow) in its Hawship lacuna and BM cells. $H \& E[A$, C\&inset of $A] \times 400$. [B\&D] $\times 100$. $[E] \times 1,000$.

In subgroup IIb, irregular immature bone was seen at margins of the defect. BM cells could be seen adjacent to these margins (Fig. 3B). The edge of the defect showed highly celluar margins formed of osteocytes in their crowded lacunae among unevenly stained matrix. Areas of irrregulaly formed bone, blood vessels and cuboidal osteo- 
blasts, were freqently noticed near bone margin (Fig. 3C). Many wide spaces separated by thin septa were frequently noticed in the defect area (Fig. 3D). Newly formed bone trabeculae were frequently seen covered with flat oesteogenic cells. Osteocytes lacunae were seen embedded in their matix. Osteoclasts were also noticed in their Hawship's lacunae on the surface of bone trabeclae. These newly formed bone trabeculae were seen surrounded with BM cells (Fig. 3E).

In subgroup IIIa, bone margins were seen irregular covered with many layers of cells. Most of these cells appeared as large cuboidal osteoblasts with intense basophilic cytoplasm and eccentric nuclei. Newly formed osteocytes could be also seen in their crowded lacunae (Fig. 4A). The graft near the margins was highly vascular. Bone trabeculae were frequently noticed towards the dura. They were covered with osteogenic cells and were characterized by homogenous (non fibrillar) acidophilic matrix. Osteocytes lacunae could also be seen embedded in their matrix (Fig. 4B). Areas of newly formed bone were noticed in the center of the defect. They appeared surrounded by flattened osteogenic cells and cuboidal osteoblasts. Dense regular highly vascular CT was also seen near the newly formed bone (Fig. 4C).

In subgroup IIIb, highly cellularized graft was seen (data not shown). Bone trabeculae covered with flattened osteo- genic cells were frequently observed at the center of the defect (Fig. 4D). The margin of the defect appeared irregular with many osteoblasts. The central part of the graft was noticed with high cellularity. Osteoblasts were seen with eccenteric rounded nuclei and negative Golgi image could be seen (Fig. 4E).

In subgroup Ia, SEM showed BM spaces filled with BM cells and lined with flat endosteal cells (Fig. 5A). Cuboidal cells mostly osteoblasts were frequently seen on the surface of the trabeculae (Fig. 5B). In subgroup $I b$, the defect area appeared filled with irregularly arranged thick fibers and extravasted RBCs. Many cells appeared with long cytoplasmic processes and were seen attached to these fibers (Fig. 5C). Subgroup IIa showed regular tissue filling areas between propylene fibers. This tissue was seen formed of regularly arranged fibers with BM cells in between (Fig. 5D). Flattened cells and cuboidal cells with cytoplasmic processes were also seen between these fibers (Fig. 5E). In subgroup IIb showed fibers of propylene mesh surrounnded by dense CT. Newly formed bone trabeculae were frequently seen in the defect (Fig. 6A). Fibers filling the defect were irrregular, the cells were interconnected and bone trabeculae were seen irregular and extending between the propylene fibers (Fig. 6B). In subgroup IIIa, osteocytes inside their lacunae with their processes were observed embedded in newly formed trabeculae (Fig. 6C).
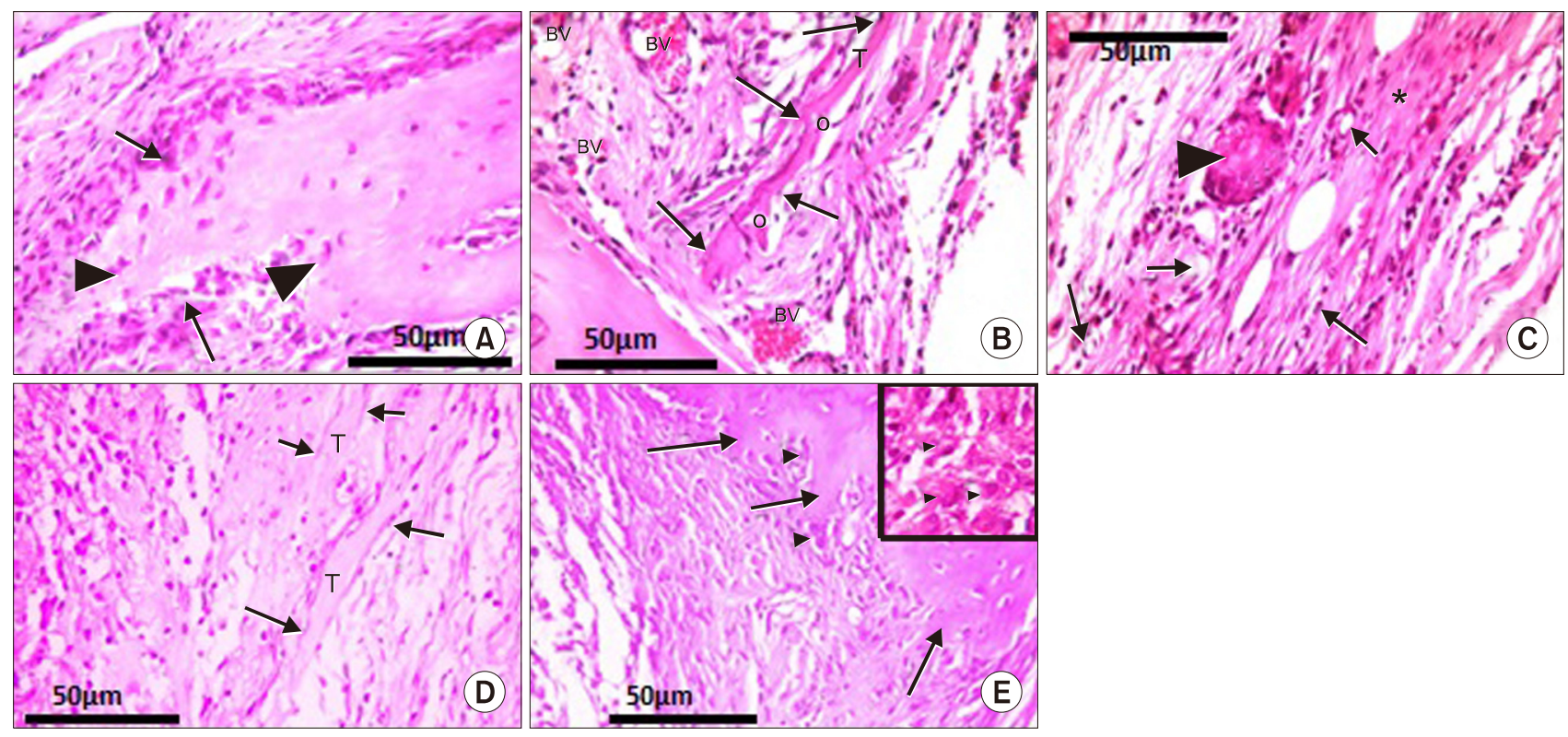

Fig. 4. (A C) IIla: (A) Highly cellular edge of defect, osteoblasts $(\uparrow$ ), newly formed osteocytes ( $\mathbf{\Delta})$. (B) Highly vascular (BV) dermal graft, bone trabecula $(\mathrm{T})$, osteocyte lacunae $(\mathrm{O})$, osteogenic cells $(\uparrow)$. (C) Center of defect: dense connective tissue $\left({ }^{*}\right)$, newly formed bone $(\boldsymbol{\Delta})$ surrounded with osteoblasts (c), osteogenic cells (f), blood vessels ( $\uparrow$ ). (D\&E) IIIb: (D) Center of defect: Bone trabeculae (T) and osteogenic cells $(\uparrow)$. (E) Irregular margin of bone defect $(\uparrow)$ and osteoblasts $(\mathbf{\Delta})$. Inset: Osteoblasts $(\mathbf{\Delta})$ and highly cellular center of defect. H\&E $(A \sim E) \times 400$. [Inset of $E] \times 1,000$. 

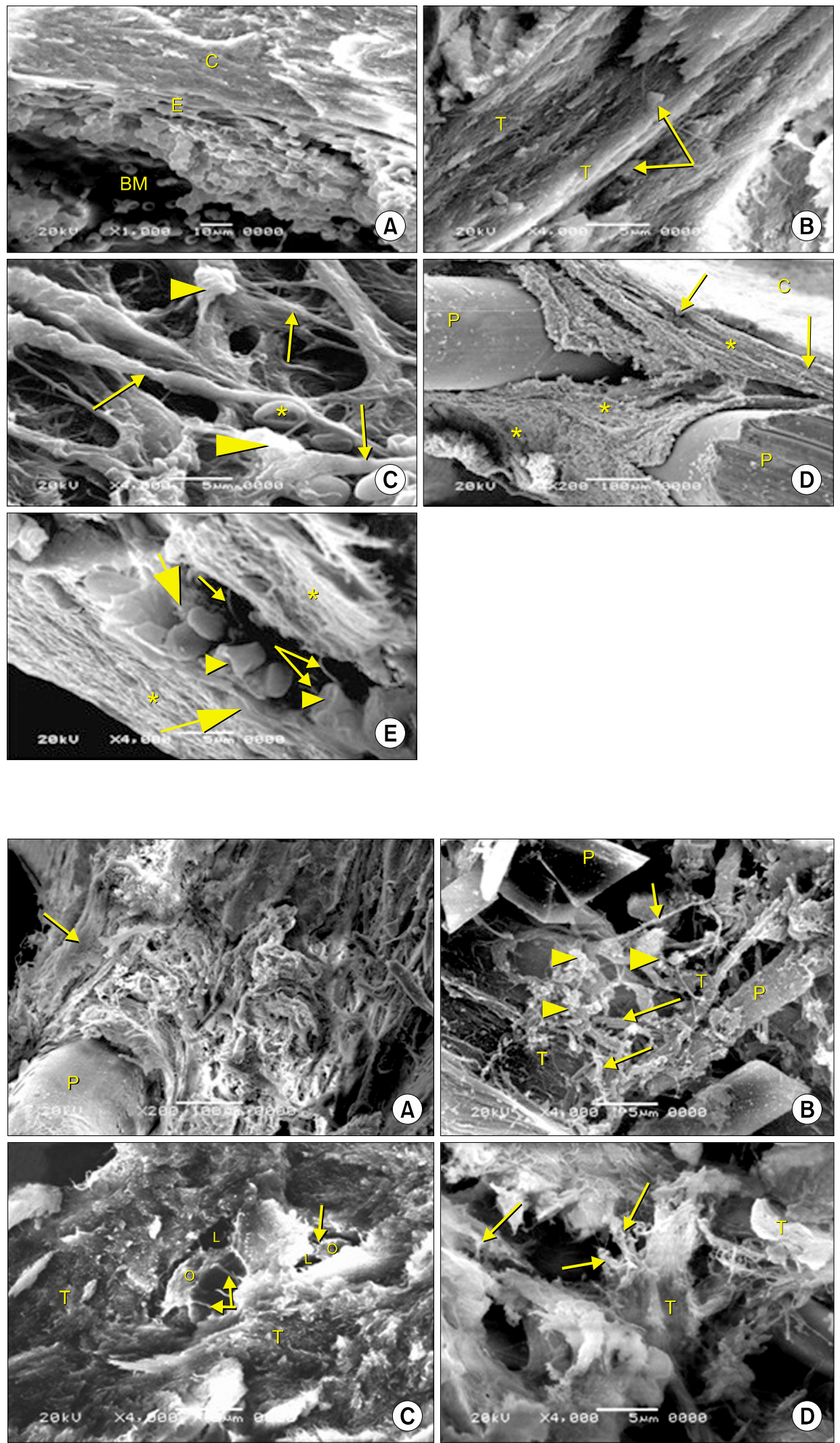

Fig. 5. Scanning electron micrographs of sagittal section of rat skull bone. (A\&B) la: (A) Clavarial bone (C) enclosing bone marrow space $(\mathrm{BM})$ is lined with flat endosteal cells (E). (B) Cuboidal bone cells $(\uparrow)$ on the surface of bone trabeculae (T). (C) Ib: Irregularly arranged collagen fibers $(\uparrow)$. Cells $(\mathbf{\Delta})$ with long processes are attached to collagen fibers and RBCs $(*)$ are seen inbetween. (D\&E) lla: (D) Fibers $\left(^{*}\right)$ and cells $(\uparrow)$ filling areas between propylene fibers (P). (C) Calvarial bone. (E) Regularly arranged fibers $\left(^{*}\right)$. Flattened cells (thick arrow) and cuboidal cells $(\mathbf{\Delta})$ with cytoplasmic processes $(\uparrow)$ are seen between these fibers. SEM $[A] \times 1,000$. [B, C\&E] $\times 4,000$. [D] $\times 200$.

Fig. 6. Scanning electron micrographs of sagittal section of rat cranial vault at the defect area. (A\&B) IIb: (A) A fiber of propylene mesh $(\mathrm{P})$, surrounnded by irregular connective tissue filling the defect. Newly formed bone trabeculae can be seen at its periphery $(\uparrow)$. (B) Irregular fibers $(\uparrow)$, interconnected cells $(\mathbf{\Delta})$ and irregular bone trabeculae $(\mathrm{T})$ are seen between propylene fibers (P). (C) IIla: Newly formed bone trabeculae $(\mathrm{T})$ and osteocytes (O) inside their lacunae (L) can be seen with their processes $(\uparrow)$. (D) IIIb: The defect is filled with connective tissue. Irregular bone trabecula $(\mathrm{T})$ and cells $(\uparrow)$ with interconnected processes can be seen between the trabeculae. SEM $[A] \times 200$. $[B, C \& D] \times 4,000$. 
Results of subgroup IIIb showed the defect area filled with CT. Irregular bone trabeculae were frequently seen in the defect. Cells with interconnected processes were noticed inbetween bone trabeculae (Fig. 6D).

\section{Immunohistochemical results}

In subgroup Ia, positive expression of osteopontin was noticed at the cement lines and in the cytoplasm of cells of $\mathrm{BM}$ and cells at the endosteum (Fig. 7A). In subgroup $I b$, minimal positive immune reaction for osteopontin was noticed in the cytoplasm of cells at the margin of the defect. Minimal reaction was also seen around blood vessels near the margin of the defect, in the dura (Fig. 7B), and in the center of the defect (data not shown).

In subgroup IIa, positive cytoplasmic expression for os- teopontin was detected in cells at the margin of the defect and on the thin rim of bone present on the inner and outer surfaces of the defect. Positive reaction was also seen in the cytoplasm of cells of the BM (Fig. 7C). While in subgroup IIb, osteopontin expression was seen in the ECM near the margin of the defect and in the cytoplasm of osteoblasts and osteocytes (Fig. 7D). In the center of the defect, prominent positive immune reaction for osteopontin was noticed in the cytoplasm of cells invading the septa that separate these BM spaces (Fig. 7E). In subgroup IIIa, intense positive cytoplasmic expression for osteopontin was noticed in the cytoplasm of cells lining the margin of the defect. In the center of the defect, the reaction was seen in the ECM (Fig. 7F). In subgroup IIIb, positive immune reaction for osteopontin was seen in the ECM near
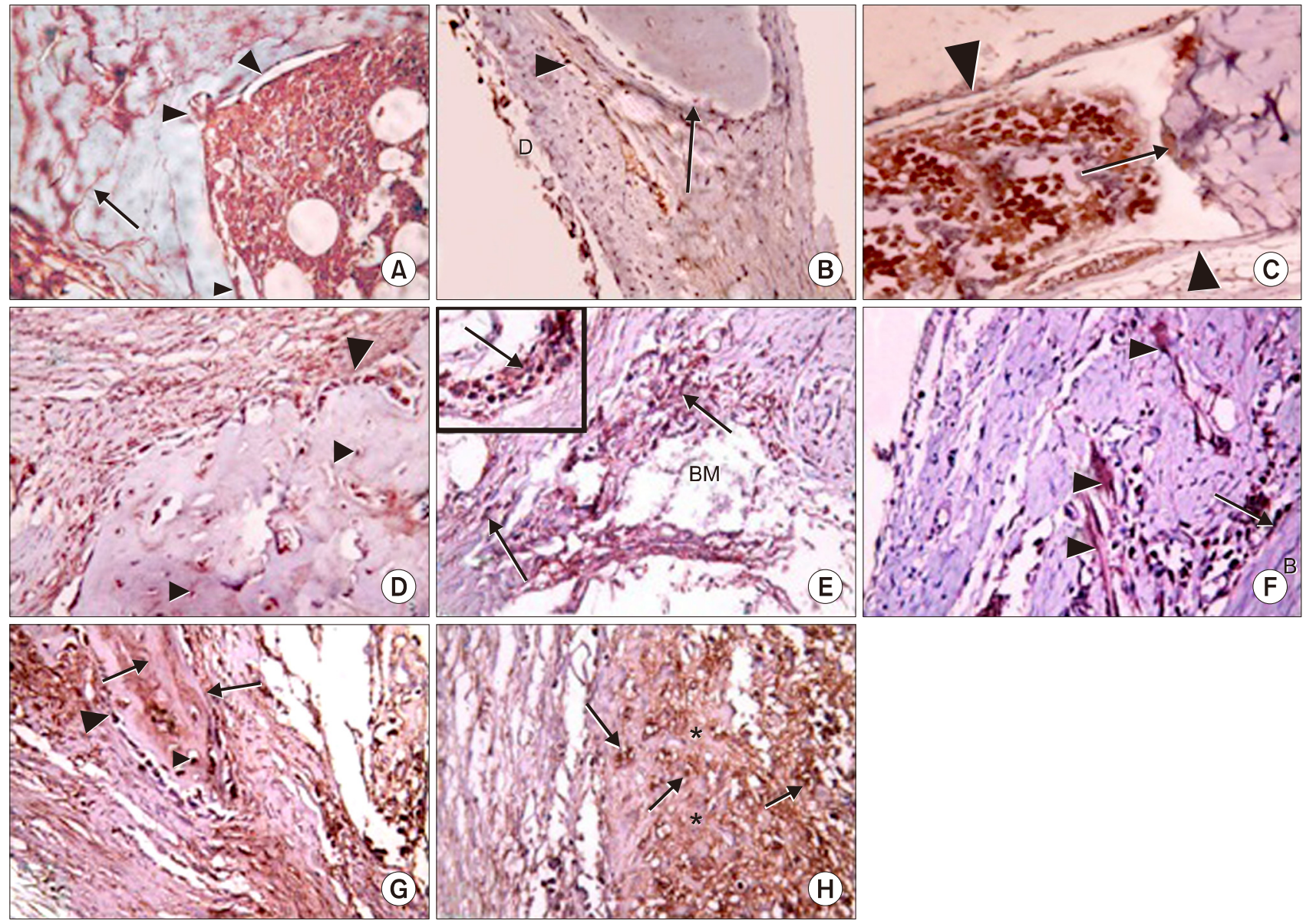

Fig. 7. (A) la: Positive immune reaction at cement lines $(\uparrow)$, in cells lining the endosteum $(\mathbf{\Lambda})$ [from above downwards: osteogenic, osteoclast, osteoblasts. (B) Ib: Minimal expression in cells lining blood vessels $(\mathbf{\Lambda})$, at margin of defect $(\uparrow)$ and dura (D). (C) Ila: Positive reaction in cells at the margin of defect $(\uparrow)$ and on thin rim of bone on the inner and outer surfaces of defect $(\mathbf{\Lambda})$. (D\&E) IIb: Positive immune reaction $(\mathrm{D})$ in osteoblasts $(\boldsymbol{\Delta})$ and osteocytes $(\mathbf{\Delta})$, (E) In cells $(\uparrow)$ surrounding newly formed bone marrow space (BM). Inset: in cells invading the septa that separate bone marrow spaces. (F) Illa: positive reaction in cells at defect margin $(\uparrow)$, in extracellular matrix $(\mathbf{\Delta})$. Clavarial bone (B). $(\mathrm{G \& H})$ IIIb: Intense reaction in $(\mathrm{G})$ : extracellular matrix, osteoblasts $(\mathbf{\Delta})$, osteocytes $(\mathbf{\Delta})$, cements lines $(\uparrow)$, $(\mathrm{H})$ in the center of the defect in cells $(\uparrow)$ and extracellular matrix $(*)$. Anti osteopontin antibody [A $\sim$ F\&its inset, G\&H] $\times 400$. 
the margin of the defect and in the cytoplasm osteoblasts and osteocytes. Cement lines were also seen with positive immune reaction (Fig. 7G). In the center of the defect, intense positive reaction for osteopontin was noticed in both cells and ECM (Fig. 7H).

\section{Histomorphometric results}

The mean percentage of bone surface covered by osteoblasts and the mean area percentage of osteopontin expression (Table 1): In subgroups Ib and IIa a significant decrease was noticed in both parameters compared to subgroups IIb, IIIa and IIIb. In subgroup IIb, a significant increase was noticed in both parameters compared to subgroups Ib, IIa and IIIa. Also a significant decrease was noticed compared to subgroup IIIb. In subgroup IIIa, a significant increase was noticed in both parameters compared to subgroups $\mathrm{Ib}$ and IIa, and a significant decrease was noticed compared to subgroups IIb and IIIb. In subgroup IIIb, a significant increase was noticed in both parameters compared to all other subgroups.

\section{Discussion}

Operative treatment for bone defect frequently requires implantation of a temporary or a permanent prosthesis, which is a challenge for orthopedic surgeons, especially in cases of large bone defects (18).

This study was carried out to assess efficacy of natural $(\mathrm{ADM})$ and synthetic (propylene mesh) scaffolds in healing of skull bone defect in the left parietal bone of adult

Table 1. Showing the mean \pm SD of the mean thickness of osteogenic layer at the margin of bone defect, the mean percentage of osteoblast surface area at the edge of the defect and the mean area percentage of positive osteopontin expression in different groups

\begin{tabular}{ccc}
\hline & $\begin{array}{c}\text { Mean percentage of bone } \\
\text { surface covered by } \\
\text { osteoblasts (\%) }\end{array}$ & $\begin{array}{c}\text { Mean area \% of } \\
\text { osteopontin expression }\end{array}$ \\
\hline Ib & $13.25 \pm 0.53^{*}$ & $1.49 \pm 0.29^{*}$ \\
Ila & $15.04 \pm 0.69^{*}$ & $2.01 \pm 0.13^{*}$ \\
Ilb & $32.51 \pm 0.37^{\triangle}$ & $7.59 \pm 0.47^{\triangle}$ \\
IIla & $20.56 \pm 0.35^{\Delta}$ & $4.39 \pm 0.39^{\wedge}$ \\
IIlb & $39.52 \pm 0.37^{\triangle}$ & $12.67 \pm 0.42^{\triangle}$ \\
\hline
\end{tabular}

*Significant decrease compared to Ilb, IIla and IIIb.

${ }^{\triangle}$ Significant increase compared to Ib, Ila, Illa.

Significant decrease from IIIb.

$\triangle$ Significant increase compared to $\mathrm{lb}$ and Ila and significant de-

crease compared to $\mathrm{IIb}$ and IIIb.

- Significant increase compared to $\mathrm{Ilb}$.

ADM: acelluar dermal graft. male albino rats. They were used either alone or seeded with stem cells.

In the current study, a bone defect of $5 \mathrm{~mm}$ diameter was done on the left parietal bone. The margins of the defects were intended to be away from skull sutures to avoid involvement of undifferentiated cells which present in the CT in the suture area as mentioned by some authors (19). In this experiment, samples were collected four weeks postoperative. It was reported that this period was enough to assess the early phase of the healing response as the stability of the materials or host reactions. However longer period of eight weeks or more might be appropriate for assessing late healing phase, as bone incorporation, resorption of materials, bone remodeling, or the amount of bone regeneration (20).

In the current study, when the defect was left to heal spontaneously (subgroup Ib), highly vascular CT was seen filling the defect area with small foci of newly formed bone in the center of the defect. This was previously observed by some authors (21). Presence of newly formed bone foci in the center of the defect in the current study was explained by some authors who mentioned that regeneration in cranial defects could occur from the cut edges of the bone or from islands of bone within the dura mater and periosteum. The periosteum provides cortical bone with blood supply. The dura contributes to the re-ossification of cranial defects due to its osteogenic potentiality (20). This could explain the presence of positive expression of osteopontin in the cytoplasm of cells at the margin of the defect and in the dura.

It was stated that osteopontin distribution at cement lines indicated bone remodeling; as cement lines were defined as being boundaries between older and newer bone. Osteopontin play a role in osteoblast adhesion and early calcification events in the cement layer (22).

On using unseeded propylene mesh in subgroup IIa, a thin acidophilic rim was seen on the inner and outer surface of the defect. Some cells were seen attached to this thin layer indicating that it might be a newly formed bone. Low bone forming activity was detected in this subgroup as there was a significant decrease in osteopontin expression compared to all groups.

After seeding of BM-MSCs on the propylene mesh (subgroup IIb), the defect area appeared divided into many spaces separated by thin septa. Irregular immature bone, bone trabeculae and foci of newly formed bone were seen near the margin of the bone. High bone forming activity was detected in this group as indicated by a significant increase in percentage of bone surface covered by osteoblasts, and the mean percentage of osteopontin 
expression.

Seeded propylene mesh appeared to give hopeful results in dealing with skull bone defects in the current study. This could be explained by other investigators who reported that the high porosity of the scaffold facilitated excellent cell infiltration and growth of blood vessels (23). It was reported that the size of the pores between fibers of propylene mesh measure about $1,000 \mu \mathrm{m}$ (24).

In the current study, ADM was a natural scaffold formed of collagen and ECM. It was used to fill the clavarial defects in group III. On using the unseeded $\mathrm{ADM}$ in subgroup IIIa, the dermal graft appeared cellular and highly vascular. Foci and trabeculae of new bone formation were seen in the central and peripheral regions of bone defect towards the dura matter. Same pattern of new bone formation was noticed by other authors on using different scaffolds (19). They stated that bone repair started from the dura side as it contained greater osteogenic activity than that of the outer periosteum, although it is well known that undifferentiated cells are present in the periosteum.

On filling the defect with seeded ADM in subgroup IIIb, the edge of the bone showed significant increase in the percentage of bone surface covered by osteoblasts, and in the mean area percent of osteopontin expression. These findings indicated highly active process of new bone formation. Strong positive expression of osteopontin was detected in the cytoplasm of osteoblasts, osteocytes and in cement lines. Distribution of osteopontin in this pattern might represent evidence for bone remodeling.

Pore size between collagen fibers differed according to the site from which skin was obtained. It was mentioned that the dermis of the skin of the back comprised loose and packed regions with heterogeneous density and inter-fibrillar spaces ranging between 1 to $20 \mu \mathrm{m}$ (25). Regarding the relation between the pore size of the scaffold and the efficacy of healing, pioneering studies were done in 2000. It showed that pore sizes less than $15 \sim 50$ $\mu \mathrm{m}$ resulted in fibrovascular in-growth, pore sizes of $50 \sim$ $150 \mu \mathrm{m}$ encouraged osteoid formation, and pore sizes greater than $150 \mathrm{~mm}$ encouraged the in-growth of mineralized bone (26). These data pushed us to unify the site of extraction of the skin used in preparation of the ADM (thin skin of the back of the rats).

It was clearly observed that seeding both scaffolds with BM-MSCs (subgroups IIb and IIIb) had led to significant increase in bone forming activity than unseeded scaffolds subgroups (subgroups IIa and IIIa). This was confirmed by statistical analysis. The integrated function between the scaffolds and the seeded cells was described by some authors. They reported that the scaffold provided the initial framework for the seeded cells to attach, proliferate, and differentiate. Then ECM created by the cells provided integrity with the new tissues over time. It was also reported that MSCs display lineage-specific differentiation when cultured on substrates that mimic the stiffness of native tissue environments. So, when MSCs were cultured on substrate that mimics the bone environment, the cells become osteogenic (22). This might explain the significant increase in the mean percentage of osteopontin expression in defects filled with seeded scaffolds in subgroups IIb and IIIB. The presence of osteopontin had traditionally been interpreted as an indicator of bone formation. It had also been reported to enhance osteoblastic differentiation and proliferation (22).

\section{Conclusions}

Tissue engineering is an ideal alternative for graft operation in healing of large bone defects. Recovery of skull bone defect was better in rats where scaffolds were seeded with BM-MSCs than rats with scaffolds alone without stem cells. During this study, the seeded ADM showed significant increase in bone forming activity as confirmed by histomorphometric and statistical results of osteoblastic surface area percent and area percentage of osteopontin expression, compared to the seeded propylene mesh groups. On the other hand both types of seeded grafts have more bone forming activity than unseeded ones.

\section{Acknowledgment}

Many thanks are due to Dr. Ahmed Mohamed Abdellah, director of experimental surgery unit, the Animal Research Center, faculty of medicine, Ain Shams University, for helping in the surgical procedure.

\section{Potential Conflict of Interest}

There are no potential conflicts of the interest or ethical problems.

\section{References}

1. Lewandrowski KU, Gresser JD, Wise DL, Trantol DJ. Bioresorbable bone graft substitutes of different osteoconductivities: a histologic evaluation of osteointegration of poly(propylene glycol-co-fumaric acid)-based cement implants in rats. Biomaterials 2000;21:757-764

2. Javaid MA, Kaartinen MT. Mesenchymal stem cell-based bone tissue engineering. International Dental Journal of Students Research 2013;1:24-35

3. Giannoudis PV, Dinopoulos H, Tsiridis E. Bone sub- 
stitutes: an update. Injury 2005;36 Suppl 3:S20-S27

4. Chatterjea A, Meijer G, van Blitterswijk C, de Boer J. Clinical application of human mesenchymal stromal cells for bone tissue engineering. Stem Cells Int 2010 doi: $10.4061 / 2010 / 215625$

5. Arnold S, Valerie J. Implant materials. In: Thorne $\mathrm{CH}$, editor. Grabb and Smith's Plastic Surgery. 6th ed. Lippincott Williams \& Wilkins, a Wolters Kluwer business; 2007. 58-65

6. Kaigler D, Pagni G, Park CH, Braun TM, Holman LA, Yi E, Tarle SA, Bartel RL, Giannobile WV. Stem cell therapy for craniofacial bone regeneration: a randomized, controlled feasibility trial. Cell Transplant 2013;22:767-777

7. Psarras S, Mavroidis M, Sanoudou D, Davos CH, Xanthou G, Varela AE, Panoutsakopoulou V, Capetanaki Y. Regulation of adverse remodelling by osteopontin in a genetic heart failure model. Eur Heart J 2012;33:1954-1963

8. Ferreira GR, Cestari TM, Granjeiro JM, Taga R. Lack of repair of rat skull critical size defect treated with bovine morphometric protein bound to microgranular bioabsorbable hydroxyapatite. Braz Dent J 2004;15:175-180

9. Spicer PP, Kretlow JD, Young S, Jansen JA, Kasper FK, Mikos AG. Evaluation of bone regeneration using the rat critical size calvarial defect. Nat Protoc 2012;7:1918-1929

10. Mokal NJ, Desai MF. Calvarial reconstruction using high-density porous polyethylene cranial hemispheres. Indian J Plast Surg 2011;44:422-431

11. Walter JB, Talbot IC. Walter and Israel general pathology. 7th ed. British Library; 1996. 184-415

12. Xu Y, Zhang G, Chang Y, Qiu YX, Wang C. The preparation of acellular dermal matrices by freeze-thawing and ultrasonication process and the evaluation of its antigenicity. Cell Biochem Biophys 2015;73:27-33

13. McFarlin K, Gao X, Liu YB, Dulchavsky DS, Kwon D, Arbab AS, Bansal M, Li Y, Chopp M, Dulchavsky SA, Gautam SC. Bone marrow-derived mesenchymal stromal cells accelerate wound healing in the rat. Wound Repair Regen 2006;14:471-478

14. Li H, Fu X, Ouyang Y, Cai C, Wang J, Sun T. Adult bone-marrow-derived mesenchymal stem cells contribute to wound healing of skin appendages. Cell Tissue Res 2006;326:725-736

15. Wang Q, Jin Y, Deng X, Liu H, Pang H, Shi P, Zhan Z. Second-harmonic generation microscopy for assessment of mesenchymal stem cell-seeded acellular dermal matrix in wound-healing. Biomaterials 2015;53:659-668

16. Suvarna K, Layton C, Bancroft J. Theory and practice of histological techniques. 7th ed. USA: Churchill Livingston; 2013.

17. Armitage P, Berry G. Statistical methods in medical research. 3rd ed. Oxford: Blackwell Scientific Publications; 1994. $12-48$

18. Dorozhkin SV. Calcium orthophosphate-containing biocomposites and hybrid biomaterials for biomedical applications. J Funct Biomater 2015;6:708-832

19. Ferreira LB, Bradaschia-Correa V, Moreira MM, Marques ND, Arana-Chavez VE. Evaluation of bone repair of critical size defects treated with simvastatin-loaded poly(lactic-coglycolic acid) microspheres in rat calvaria. J Biomater Appl 2015;29:965-976

20. Sohn JY, Park JC, Um YJ, Jung UW, Kim CS, Cho KS, Choi SH. Spontaneous healing capacity of rabbit cranial defects of various sizes. J Periodontal Implant Sci 2010; 40:180-187

21. Claes L, Recknagel S, Ignatius A. Fracture healing under healthy and inflammatory conditions. Nat Rev Rheumatol 2012;8:133-143

22. Galindo P, Hernndez P, Padial M, Luisa M, Crespo V, O'Valle F. Osteopontin expression in an organic bovine bone Immunohistochemical osteopontin expression in bone xenograft in clinical series of maxillary sinus lift. J of Oral Sci \& Rehab 2015;1:42-50

23. Cunniffe GM, Díaz-Payno PJ, Ramey JS, Mahon OR, Dunne A, Thompson EM, O'Brien FJ, Kelly DJ. Growth plate extracellular matrix-derived scaffolds for large bone defect healing. Eur Cell Mater 2017;33:130-142

24. Zogbi L. The use of biomaterials to treat abdominal hernias. Biomaterials Applications for Nanomedicine, Prof. Rosario Pignatello (Ed.), ISBN: 978-953-307-661-4, InTech; 2011. 359-382

25. Wolf K, Alexander S, Schacht V, Coussens LM, von Andrian UH, van Rheenen J, Deryugina E, Friedl P. Collagen-based cell migration models in vitro and in vivo. Semin Cell Dev Biol 2009;20:931-941

26. Petite H, Viateau V, Bensaï W, Meunier A, de Pollak C, Bourguignon M, Oudina K, Sedel L, Guillemin G. Tissueengineered bone regeneration. Nat Biotechnol 2000;18: 959-963 